\title{
Experiences of Weight Stigmatization: A Review of Self-Report Assessment Measures
}

\author{
Jenny A. DePierre Rebecca M. Puhl \\ Rudd Center for Food Policy and Obesity, Yale University, New Haven, CT, USA
}

\author{
Key Words \\ Weight stigma $\cdot$ Victimization $\cdot$ Obesity $\cdot$ Assessment $\cdot$ Review
}

\begin{abstract}
Background: Overweight and obese persons are vulnerable to frequent stigmatization and discrimination because of their weight. Despite widespread prejudice towards obese persons, many questions remain regarding the nature, extent, and impact of weight-based stigmatization experienced by so many people. However, improving our knowledge in this area will only be as good as the measures we have to study this phenomenon. Our paper is the first to provide a comprehensive summary of published quantitative self-report measures available for assessing experiences of weight stigmatization in children and adults. Methods: This review examined 22 measures of self-reported weight stigmatization and highlights the strengths and limitations of existing assessment. Results: Existing measures contain a number of limitations and have been assessed in samples lacking diversity. Improvements in measurement are needed to achieve a clearer understanding of the nature and extent of self-perceived weight stigmatization and to develop measures that accurately reflect this type of stigmatization. Conclusions: Specific directions for future research that will help improve measurement of self-perceived weight stigmatization and advance this area of study are highlighted.
\end{abstract}

Copyright (c) 2012 S. Karger GmbH, Freiburg

\section{Introduction}

As the prevalence of obesity has increased, so has research attention to its social consequences. During the past decade there has been a steady increase in research examining bias, stigma, and discrimination towards individuals who are overweight or obese [1]. This 
body of literature has identified multiple sources of weight stigmatization toward obese persons, including negative stereotypes and prejudice endorsed by employers, health care providers, educators, peers, family members, and the media [1, 2]. The consistency of findings in this field may reflect the social acceptability of weight bias in many Westernized societies [3]. Indeed, recent studies indicate a 66\% increase in the prevalence of weight discrimination over the past decade in the USA [4]. Emerging research has documented numerous adverse psychological and physical health outcomes among obese children and adults who have been stigmatized because of their weight, including risk of depression, anxiety, low self-esteem, poor body image, suicidal ideation, maladaptive weight control practices, binge eating, and avoidance of physical activity [5-8].

Despite considerable work in this area and the pervasiveness of this problem, methods to assess experiences of weight stigmatization among obese individuals have been inconsistent and generally lacking in the literature. Instead, most research to date has focused on the stigmatizer's perspective (e.g., attitudes and behaviors) toward obese persons, rather than the target's experiences of stigmatization. For example, numerous studies have assessed prejudiced hiring practices by employers against obese employees, stigmatizing attitudes by health care providers toward obese patients, or negative stereotypes endorsed by peers and family members toward obese children and adults [1,9-12]. Ruggs and colleagues [13] suggest that research examining the stigmatizer's perspective has dominated the literature because it is easier to conduct than assessing personal experiences of weight stigmatization among obese persons; namely, researchers can simply ask participants about their attitudes toward obese persons, or show participants images or videos of obese targets, and few (if any) obese persons are needed to conduct this research. Additionally, there is a tendency for research to initially focus on institutional and societal forms of bias, as has been seen in the case of racism, before turning its attention to the effect of perceived stigmatization on the individual.

Documenting the stigmatizer's perspective has been informative and has improved our understanding of the nature and extent of weight stigmatization, but there are important research questions that have not been addressed because of inadequate assessment of the target's perspective. For example, little is known about the frequency and nature of specific forms of verbal, physical, cyber, or relational weight stigmatization experienced by obese individuals across different settings, or how obese children and adults react to these different experiences. A primary reason for existing gaps in knowledge is the lack of consistent, comprehensive measures available to assess experiences of, and reactions to, weight-based stigmatization among overweight and obese youth and adults. To date, many studies have assessed weight-based stigmatization or discrimination with only one or two general questions about previous history of teasing (e.g., 'Do others tease, joke, or make fun of you because of your weight?' [14], or 'Have you ever experienced weight discrimination, or had a negative experience because of your weight?' [15]) or have used general measures of teasing or discrimination (not specific to weight) in obese samples [16]. These simply examine correlations between BMI and general teasing rather than asking individuals about their experiences of stigma and discrimination specifically related to their body weight. While some measures have been developed in recent years to provide a more direct assessment of experiences with weight stigma, these tend to focus on only one type of stigma (e.g. verbal comments and teasing), seldom differentiate between different perpetrators or settings of stigma, and have not been consistently used in the literature.

To obtain an accurate understanding of the nature, extent, and impact of experiencing weight stigmatization, there is a need for measures that provide a comprehensive assessment of the frequency and range of weight-based stereotypes, prejudice, and discrimination experienced from multiple sources in multiple settings. Comprehensive assessment of the target's 
perspective is critical in order to obtain an accurate understanding of the significant challenges facing obese persons, to inform and guide anti-bullying and stigma reduction interventions, and to improve support channels that can help obese youth and adults to adaptively cope with the consequences of weight-based stigma and prejudice. Thus, the aim of this paper is to provide a summary of existing survey measures in the published literature that assess experiences of weight-based teasing, stigmatization, and discrimination. Specifically, this review examines existing quantitative measures that assess experiences of weight stigmatization from the target's perspective, summarizes the strengths and weaknesses of these measures, and offers recommendations for improving assessment of experiences of weight stigmatization.

\section{Methods}

A systematic literature search was conducted for studies using measures examining weight stigma, discrimination, or weight-related victimization in any form, in adult and youth samples. For the purpose of this review, we defined weight stigma as pertaining to any type of perceived negative bias or stereotyping because of one's excess weight, including verbal (e.g., insults about weight), physical (e.g., physically attacked), relational (e.g., being avoided or excluded), and environmental stigma (e.g., not being able to fit into (or being charged extra fees for) seats on public transportation). Discrimination was defined more specifically to include systemic prejudice and unfair treatment of an individual because of their excess weight (e.g., not being hired for a job because of one's body weight). Finally, weight-related victimization was conceptualized as acts and/or threats of verbal teasing, cyber bullying, physical aggression (e.g., being pushed, shoved), or relational victimization (e.g., being excluded from activities) because of excess weight, which are most often studied in the context of youth experiences of weight stigma.

A computer-based search of published studies was conducted between May and December 2011 using scientific research databases including PsycInfo, PubMed, and the online academic search engine Google Scholar. Key search terms used in all databases included 'weight', 'body weight', 'obesity', 'overweight', 'stigma', 'bias', 'prejudice', 'teasing', 'bullying', 'discrimination', 'victimization', and 'stereotype(s)'. These search terms were combined with singular and plural terms for assessment methods including 'assessment', 'scale', 'measure', 'instrument', 'inventory', 'survey', 'meta-analysis' and 'psychometric(s)' in a variety of combinations. No limits were placed on language, country of origin, or year of publication. This yielded 869 articles from PsychInfo and 1,630 articles from PubMed. Additionally, supplementary searches of book chapters and the references lists of selected articles as well as of Google Scholar were also conducted to locate any additional related studies. The whole search yielded 33 studies. Measures from these studies were retained if the scale in question had been used to directly assess weight bias, self-stigma, or discrimination based on body weight in any form, or included a subscale which assessed one or more of these constructs. This included measures of general discrimination and stigma that had been modified to assess stigma specifically related to body weight. However, measures that assessed more general appearance-related stigma without specifying body weight were excluded (for a review of more general measures of appearance-based stigmatization, see Menzel et al. [17]). In addition, exclusions were made if i) measures were shorter than 3 items or were longer measures but used fewer than three items to assess weight stigmatization, ii) measures included only items with dichotomous response options (e.g., 'yes' versus ' no') which did not form a meaningful scale (these were most often shorter than three items), or iii) measures were in unpublished manuscripts and dissertations. Measures that 


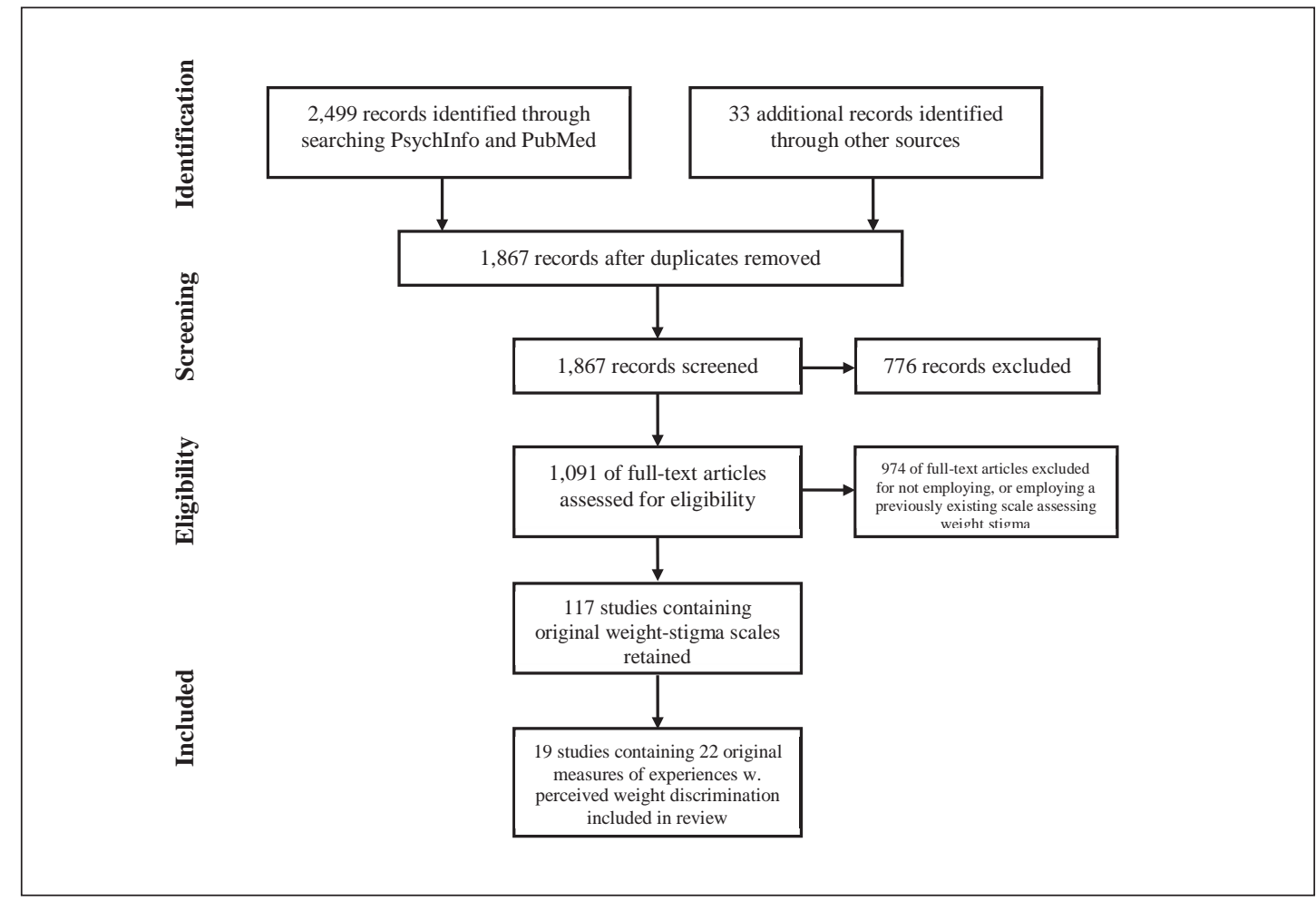

Fig. 1. Measure selection.

had been replicated or tested in modified formats that significantly changed the constructs tested (e.g. the source or setting of experienced weight stigma) were retained, and these altered surveys were included as separate measures. See figure 1 for a summary of the selection process.

This refined search process yielded 117 distinct original or modified survey measures of weight stigma. Several additional exclusions were made to ensure that the final sample consisted only of quantitative measures assessing experiences of weight stigmatization. First, 6 measures were excluded that assessed the construct of self-stigmatization (e.g., selfblame and internalization of weight stigmatization), 11 were excluded because they were semi-structured, mixed qualitative and quantitative measures of weight stigma with fewer than 3 items quantitatively assessing experiences with weight stigma, and 9 were excluded because they indirectly measured weight bias through the rating of visual stimuli. In addition, 58 measures were quantitative measures that assessed the stigmatizer's perspective (e.g., stigmatizing attitudes toward obese individuals). These measures were excluded because the stigmatizer's perspective is not the focus of this review, and this literature has been recently summarized elsewhere [13]. This left 33 measures that directly assessed personal experiences of weight-based stigma and/or discrimination, including weight-related teasing. Of these, exclusions were made for one measure that included multiple physical attributes (one of which was body weight) but did not distinguish between different forms of appearance stigma [18], and three scales which had been adapted from previous measures but did not significantly change the construct measured [19-21]. Similarly, seven scales which assessed appearance-related commentary, including some items related to weight, (e.g., 'My parents told me to eat different foods in order to lose weight or 
keep from gaining weight'[22]) were evaluated by the authors as sufficiently different constructs from measures of weight-based discrimination or stigmatization, and were also excluded from the final analysis. The remaining 22 measures were retained as the final sample for the present review. An additional search for articles that cited the original study for each of these 22 measures was conducted, and from this search, references of other studies employing each measure were compiled.

\section{Results}

Tables 1 and 2 describe and summarize basic psychometric characteristics of the 22 measures assessing experiences of weight stigmatization where original studies are listed by the first author. Table 1 summarizes measures that assess weight stigmatization in adults, and table 2 presents measures that assess weight stigmatization in youth. The numbers used to index the measures in this chart will be used throughout the paper. Of the 22 measures, 14 assessed experiences of weight stigmatization in adults (measures \#1-11, 13-15) [23-36] seven assessed experiences of weight stigmatization in youth (\#16-22) [37-43], and one measure has been consistently used to assess weight stigmatization in both children and adults (\#12). The latter is listed with the adult measures in table 1, as it was originally developed for use in an adult sample. 21 measures were developed by researchers in the USA, and one measure was developed by researchers in the UK (\#4). All measures assessing weight stigmatization were constructed with Likert rating scales, including two measures that used a combination of Likert scales, free-response questions, and categorical items (\#20, \#21).

\section{Body Weight Distribution of Samples}

Given that the focus of measurement is experiences of weight stigmatization, it is important to examine the body weight distribution of samples in which the measures were originally assessed. Of the adult measures, four studies employed samples comprised entirely of overweight or obese adults ( $\# 5, \# 9, \# 10, \# 15)$, three studies contained samples with at least $50 \%$ overweight or obese adults (\#3,\#6,\#7), three studies contained samples with a majority of normal-weight participants (\#1, \#4,\#8), and three studies reported no description of body weight of participants (\#2, \#11, \#12). For two measures (tested using the same sample), BMI was calculated, but not reported (\#13, \#14). For those studies reporting weight distribution of participants, all used the classification of overweight and obesity in adults by the National Heart, Lung and Blood Institute of the National Institutes of Health [44], with the exception of two studies (\#7, \#10) which did not use these specific classifications but reported the BMI range of participants.

Among the measures assessing weight stigmatization in youth, one study employed a sample comprised entirely of overweight or obese youth (\#22), one study contained a sample with at least $50 \%$ overweight or obese youth (\#17), one study contained a sample with a majority of normal-weight youth (\#20), two studies provided a total sample mean BMI in the normal-weight range (\#16, \#18), and two studies reported no description of body weight of participants (\#19, \#21). Of the studies reporting weight distribution in youth participants, BMI percentiles were calculated using age and gender, but classifications of overweight and obese differed across studies. One defined youth 'at risk for overweight' (BMI = 85th-95th percentile) and overweight/obese (BMI = $\geq 95$ th percentile) (\#17), another study classified overweight at $\geq 85$ th percentile and obesity as $\geq 95$ th percentile (\#20), and another classified overweight as 90th-99th percentile, and obesity as $\geq 99$ th percentile (\#22). 

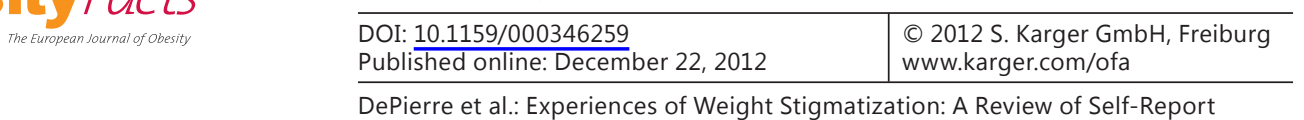

DePierre et al.: Experiences of Weight Stigmatization: A Review of Self-Report Assessment Measures
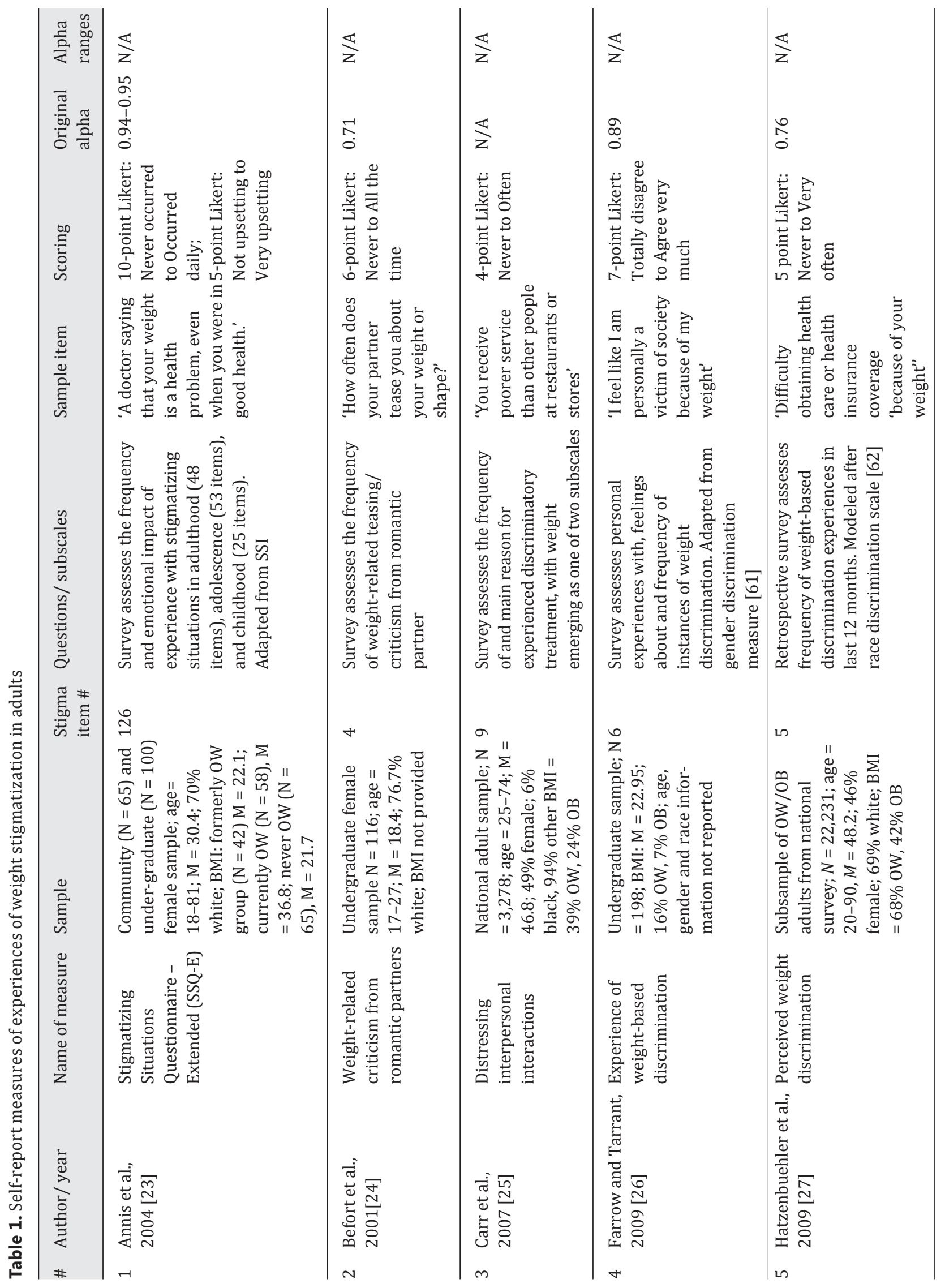

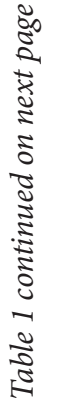


ty

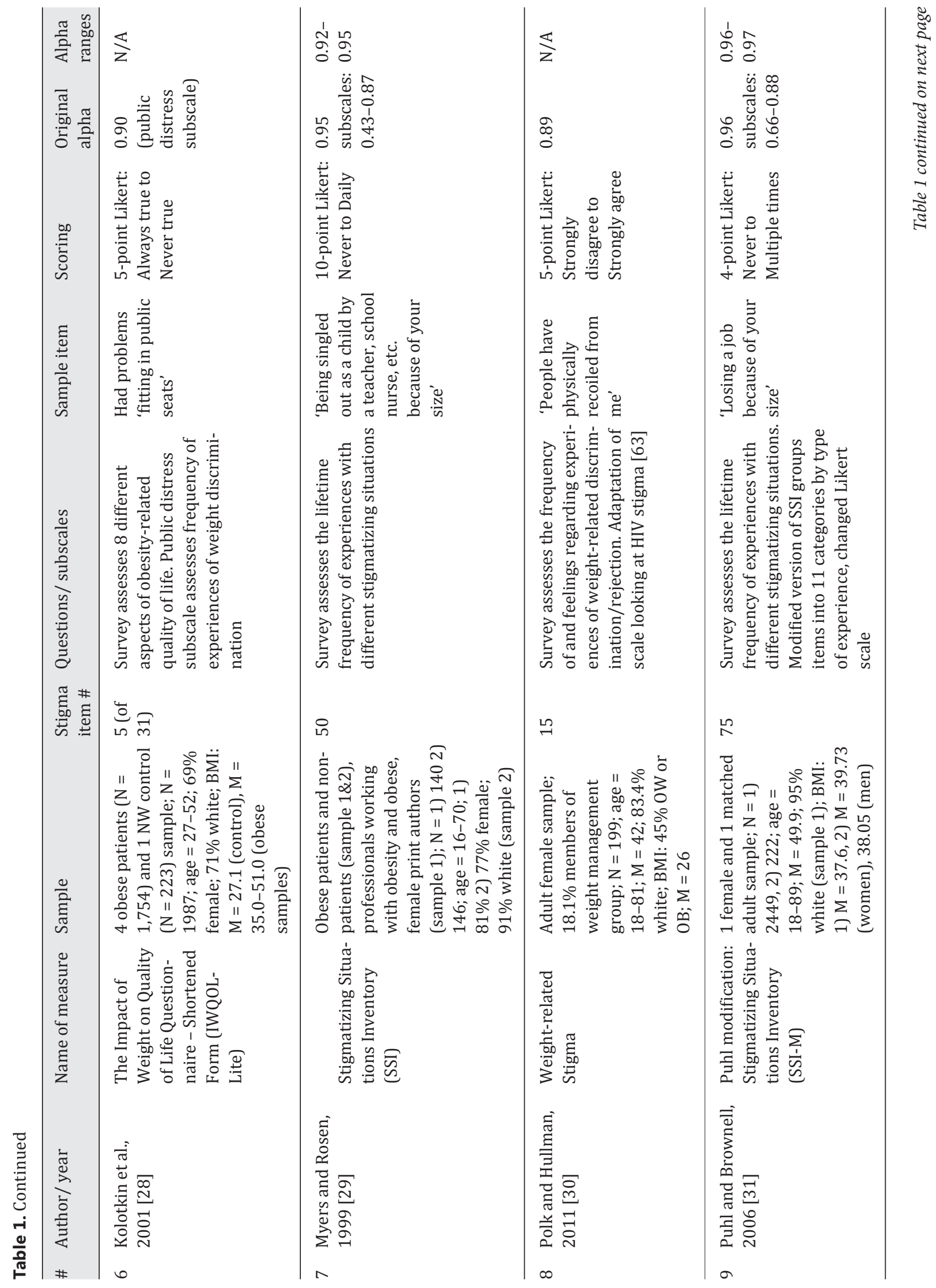




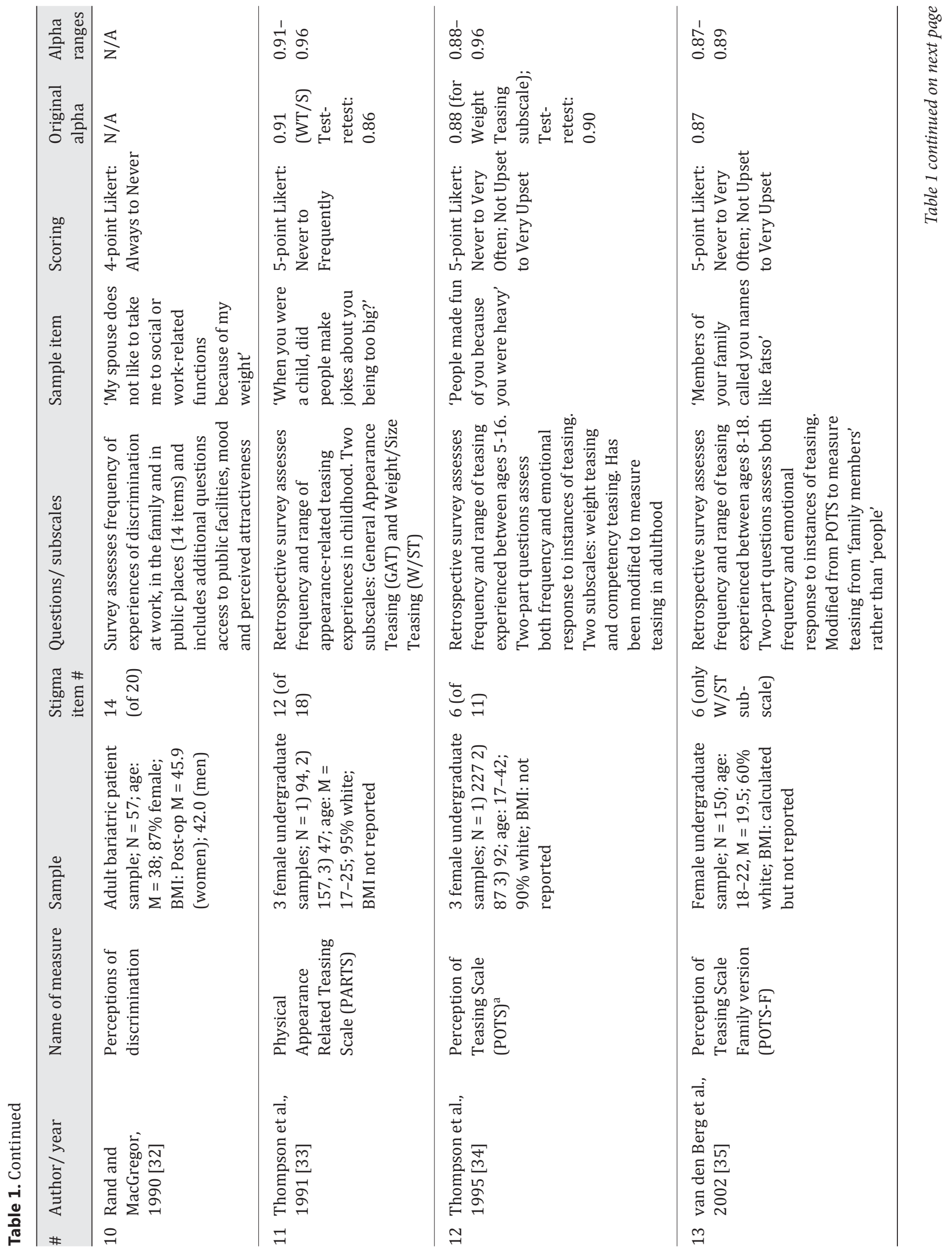




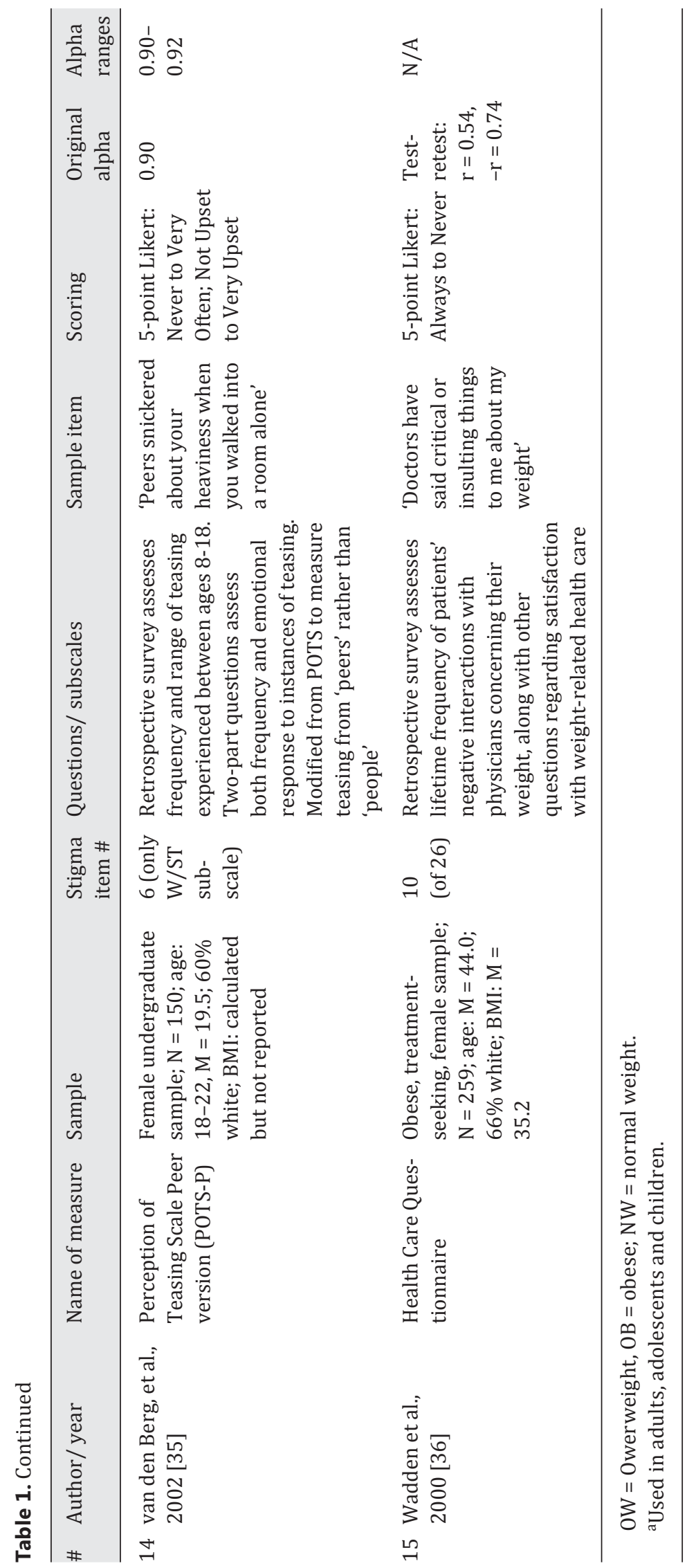




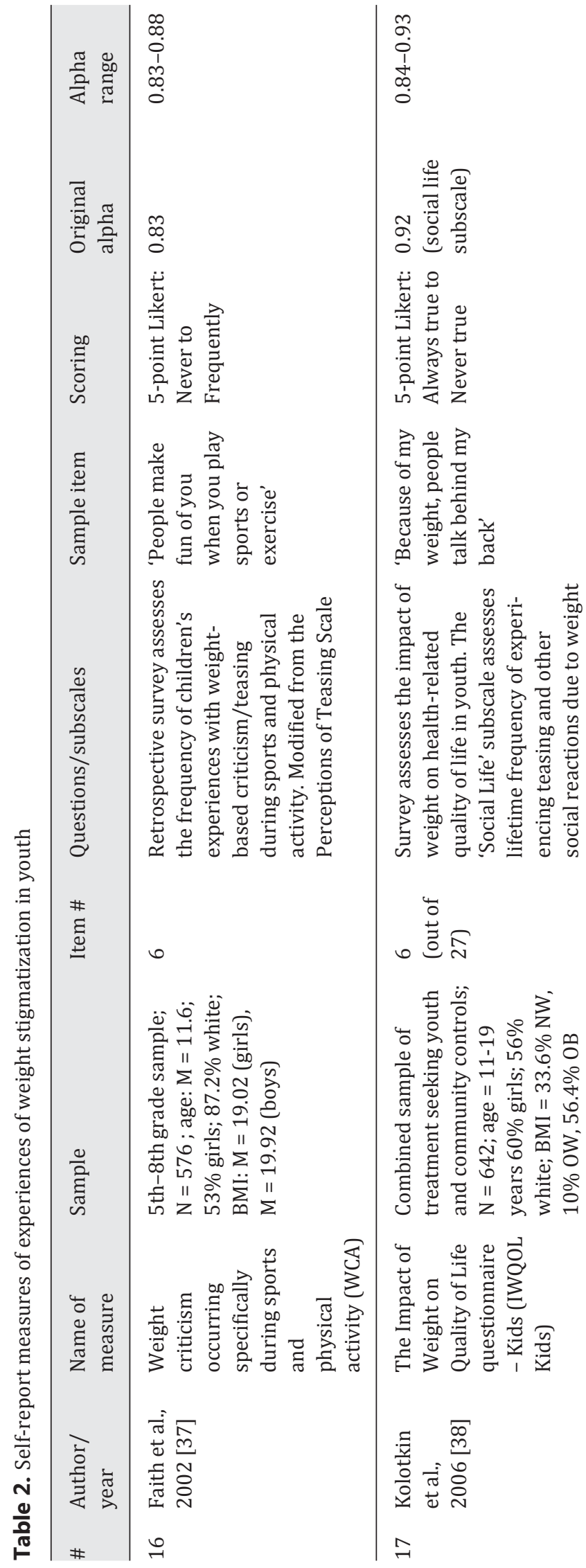

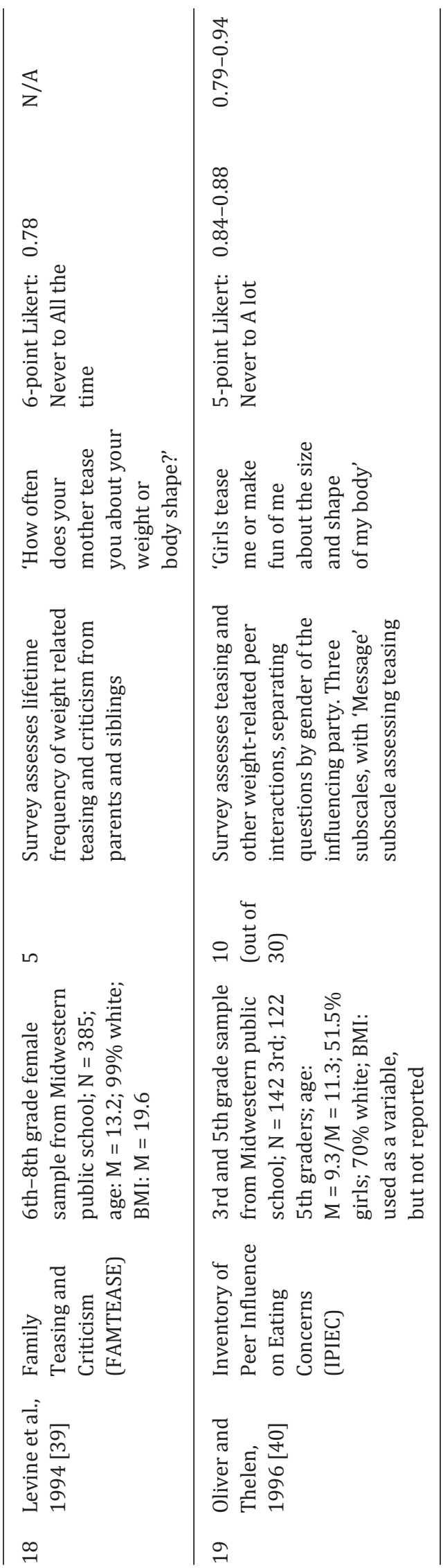

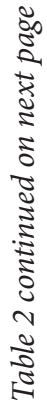


DePierre et al.: Experiences of Weight Stigmatization: A Review of Self-Report Assessment Measures

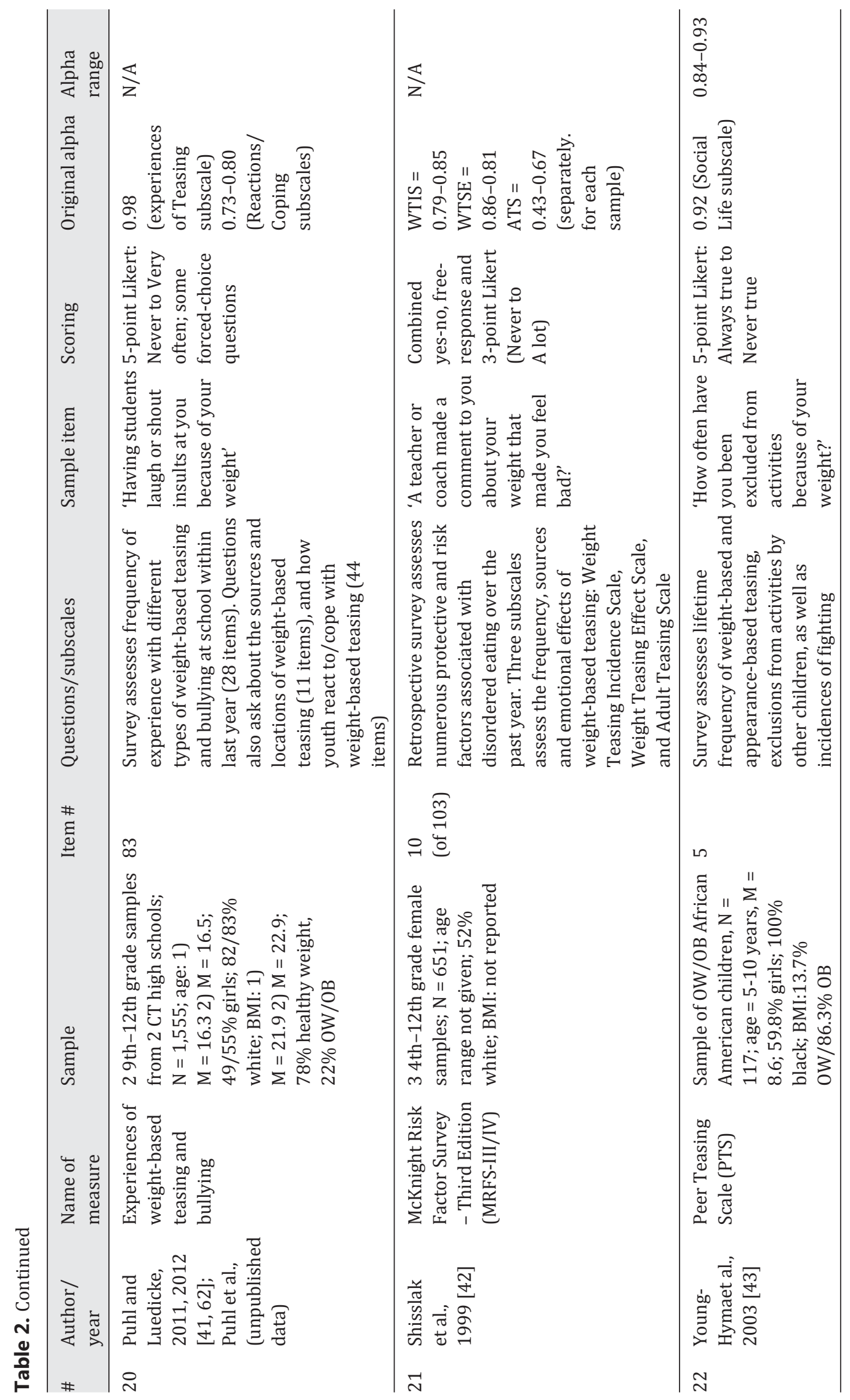




\section{Gender and Race of Samples}

A slight majority of measures were assessed in samples of both females and males. Among the adult samples, seven measures were tested with both women and men (\#3-7, \#9, $\# 10$ ) and eight measures were tested only with women (\#1,\#2, \#8,\#11-15). Among measures assessing youth, five were tested with both boys and girls (\#16, \#17, \#19, \#20, \#22), and two were tested only with girls (\#18, \#21). The racial composition of samples across studies was primarily Caucasian, including five studies where samples were over 90\% Caucasian (\#7, \#9, $\# 11, \# 12$, \#18). Among adult measures, all samples contained a majority of Caucasian participants, with the exception of two studies in which race was not reported (\#4, \#10). Similarly, all youth measures contained primarily Caucasian samples with the exception of one (\#22), which contained $100 \%$ African-American participants.

\section{Characteristics of Measures}

\section{Internal Consistency}

19 of the 22 measures included assessments of internal consistency. Eight scales had a high Cronbach's $\alpha$ of above 0.90 (\#6, \#7, \# 9, \#11, \#14, \#17, \#19,\#20), six scales had Cronbach's $\alpha$ between 0.80 and 0.90 (\#4, \#8,\#12,\#13,\#16, \#21), and five scales had Cronbach's $\alpha$ between 0.70 and 0.80 (\#2,\#5, \#18, \#22). Only one measure had a weight discrimination subscale with a Cronbach's $\alpha$ of less than $0.70(\alpha=0.65)(\# 21)$, although the average Cronbach's $\alpha$ of the weight-related subscales on this measure was between 0.70 and $0.80(\alpha=$ $0.73)$. Additionally, 3 studies did not report Cronbach's $\alpha$ (\#3, \#10, \#15). For modified scales (\#1-5, \#8, \#9, \#12-14, \#16), internal reliability, where reported, was similar to that of the original measures (tables 1 and 2).

In order to further assess internal consistency, searches were conducted to identify any studies that employed the measures in different samples. If a scale had been used beyond the original study, and Cronbach's $\alpha$ were available for the citing articles, Cronbach's $\alpha$ ranges are presented to demonstrate the variability in internal consistency across different samples (tables 1 and 2). If a measure was very frequently cited, ten studies using the scale were selected at random in order to calculate this alpha range.

Ten adult measures (\#2, \#3, \#6, \#7, \#9, \#11-15) and 4 child measures (\#16, \#17, \#19, $\# 21$ ) had been used in full or comparable form in at least one other sample. Cronbach's $\alpha$ ranges obtained for these scales, where available, were similar to those from the original studies (tables 1 and 2 for reported Cronbach's $\alpha$ of each measure).

\section{Survey Length}

Most of the measures included in this analysis are of moderate length. All but four of the measures contain 20 items or fewer related to weight-based victimization. Five of the scales contain five or fewer items (\#2,\#5, \#6, \#18,\#22), 10 contain between 6 and 10 items (\#3, $\# 4, \# 12-17, \# 19, \# 21)$, three contain between 11 and 20 items (\#8, \#10, \#11), and four measures are longer than 20 items (\#1, \#7, \#9, \#20). Notably, all four of the longest measures contain 50 or more items $(50,75,83$, or 126$)$.

\section{Intended Purpose of Measures}

Although all of the measures included in this analysis assess experiences of weight stigmatization, there is variability in the language used to describe the central constructs that the survey items were designed to assess. For example, descriptors of stigmatizing experiences included 'discrimination', 'stigma', 'teasing', 'criticism', 'bullying', and other 
labels. Among the measures assessing adult experiences, four measures specifically assessed weight-based 'discrimination' and 'discriminatory treatment' based on body weight (\#3-5, \#10), and four measures assessed weight-based 'stigma' or 'stigmatizing experiences' (\#1, \#7-9), including one measure which also assessed self-stigma (\#8). In addition, three measures assessed weight-related teasing (\#13,\#14) and criticism (\#2), and two measures were designed to assess general (\#12) and physical/body appearance (\#11) teasing (e.g., 'Did other kids ever make fun jokes about your hair?') but included subscales to assess weight-based teasing. Finally, two of the adult measures (\#6, \#15) did not include weight-based stigmatization as the primary focus, but included a subscale that assessed stigma-based items. For example, the Impact of Weight on Quality of Life Questionnaire Shortened Form (IWQOL-Lite) was designed to assess the impact of weight on quality of life (\#6), but included a subscale on experiencing distress because of weight including public ridicule and discrimination. The Health Care Questionnaire (\#15) was designed to assess how satisfied treatment-seeking obese patients were with the care provided by their physicians, but included a subscale on past stigmatizing weight-related interactions with their physicians.

For measures assessing youth experiences of weight stigmatization, surveys most often assessed these experiences in the context of being teased or bullied because of weight. In particular, three measures assessed specific teasing experiences related to weight (\#18, $\# 20$, \#22). One of these measures assessed bullying as well as teasing experiences (\#20), another included both teasing and weight-based 'criticism' from others (\#18), and the third focused on verbal, non-verbal and physical weight-related victimization, but also included items assessing general appearance teasing (\#22). A fourth measure focused entirely on weight-based criticism in the context of physical activity settings (\#16). The remaining three youth-oriented measures (\# 17, \#19, \#21) were intended to assess other constructs, such as health-related quality of life (\#17), peer influences on eating concerns (\#19), and risk factors associated with disordered eating (\#21) but include items or subscales on weightbased teasing from different sources.

\section{Use of Weight Terminology}

The use of terminology to describe body weight is another important characteristic to examine in measures of weight stigmatization, as different terminology determines the target population that can be sampled, such as whether experiences of weight stigmatization can be assessed among samples with a range of body weight categories, or are intended for use only with samples of overweight and/or obese respondents. For example, measures that assess experiences of stigmatization based on one's 'weight' (e.g., 'Have you ever been teased because of your weight?') can be used with individuals across weight categories (e.g., underweight, normal weight, overweight, obese), whereas measures that phrase questions according to stigmatization because of excess weight (e.g., being 'overweight') will only be appropriate for use among overweight or obese respondents.

There is considerable variation in weight-based terminology used across existing measures to assess weight stigmatization. Among adult measures, four measures use 'weight' as the only descriptor (\#5, \#6,\#8,\#10), several use both 'weight' and a variety of descriptors such as 'heavy', 'fat' or 'overweight' (\#1,\#2, \#4, \#7, \#9, \#15), one measure uses 'weight and height' (\#3), another measure specifically assesses stigma experienced for being 'overweight' (\#11), and three primarily use the term 'heavy' (\#12-14). Among youth measures, 'weight' is used as the only descriptor in four measures (\#17, \#20-22), 'weight or body shape' (\#18) and 'heavy' (\#16) are each used in one measure, and 'fat' and 'size' are used in another measure (\#19). Thus, depending on whether one's intention is to examine experiences of weight stigmatization specifically among overweight or obese persons, or 
across a broader range of weight categories, careful attention should be given to the weight terminology used prior to selecting appropriate measures.

Time Period of Assessment

Across both adult and youth measures, there was variability in assessment of the period of time that weight stigmatization was experienced. Among adult measures, three scales assessed experiences of weight stigmatization on a day-to-day basis (\#2, \#3, \#10), one measure assessed experiences of weight stigmatization during the previous 12 months (\#5), six measures assessed lifetime history of stigmatization (\#4, \#6-9, \#15), four measures assessed recollections of weight stigmatization during childhood (\#11-14), and one measured history of discrimination in childhood, adolescence and adulthood (\#1). Among youth measures, four scales assessed lifetime experiences of weight stigmatization (\#17-19, \#22), two assessed weight stigmatization during the previous 12 months (\#20, \#21), and one assessed weight-based criticism on a day-to-day basis (\#16).

Frequency

In addition to knowing if a person has experienced weight stigma, it is also important to assess how frequently this has occurred, particularly given research on racial discrimination which indicates that chronic stressors may be more predictive of health than acute ones [45]. Twenty of the 22 measures assessed frequency of stigma experiences from 'never' to 'often/daily/frequently' or, for three measures, 'never' to 'always'. Two of the adult measures (\#4, \#8) included statements such as 'Prejudice against overweight people has affected me personally' (\#4) and 'People have told me I just need to have more discipline to change my appearance' (\#8), which were rated on spectrums from agree to disagree.

\section{Types of Weight Stigmatization}

Given the diverse settings in which weight stigmatization has been documented $[1,2]$, it is important to examine the types of weight stigmatization that current measures assess. Among adult measures, all contain items assessing weight stigmatization in at least one specific setting (e.g., the workplace, public spaces, health care, etc.), but only four of these measures contain multiple questions (e.g., at least three items per setting) assessing experiences of weight stigmatization in public, in relationships with family and friends, in health care, in the workplace, and at school (\#1,\#7,\#9, \#10), one of which also subcategorizes scale items according to specific settings that could be used as distinct subscales (\#9). One measure specifically assesses stigmatization that takes place in a health care setting (\#15). In contrast, among measures assessing general or physical appearance teasing with subscales on weight-related experiences, (\#11,\#12), as well as in those that focus exclusively on weight teasing $(\# 2, \# 13,14)$, items primarily assess weight-related feedback or criticism (e.g., 'People called you names like 'fatso"' (\#12)), most of which are not anchored to particular settings.

Among youth-oriented measures, five scales assessed weight stigmatization in various (e.g., school, home, with peers) or unspecified settings (\#17-19, \#21, \#22), one measure assessed weight-based criticism specifically in the context of physical activity settings (\#16), and another measure assessed weight-based bullying in various locations specifically on school campus (\#20).

\section{Sources of Weight Stigmatization}

While individuals can experience weight stigmatization from numerous types of individuals in their lives (e.g., family members, teachers, health care providers), existing measures differ according to whether or not (or how many) specific sources of weight stig- 
matization are assessed. For example, 11 adult measures primarily included items that assess general weight-stigmatization from anyone (e.g., questions that ask respondents whether they have ever been called derogatory names because of their weight, but do not specify the source or the perpetrator). Of these, 5 included only such questions (\#3, \#4, \#6, $\# 8, \# 12$ ). One measure specifically assesses teasing and criticism from romantic partners (\#2), one examines negative interactions with physicians (\#15), and two examine weightrelated teasing specifically from family members (\#13) and peers (\#14). Three measures (\#1, \#7, \#9) assess a broad range of sources of weight stigmatization (e.g., family members, romantic partners, strangers, peers). One measure assesses stigmatization from family members and peers as well as from 'people' in general (\#11), and one includes one question specific to weight stigmatization from health care providers (\#5). In addition, two measures include questions about environmental stigma without a perpetrator, such as being unable to fit through aisles or use public transportation because of one's size (\#6, \#10).

Among youth measures, two assess general (unnamed) sources of stigmatization (\#16, $\# 17)$, three assess weight teasing and bullying from peers (\#19, \#20,\#22), one measure assesses teasing from family members (\#18), and one measure assesses a variety of sources of weight stigmatization (peers, family members, teachers) (\#21).

\section{Impact of Stigma}

In addition to assessing the occurrence of self-reported weight-related stigmatization, for studies aiming to examine the consequences of such experiences, it may be informative to measure its severity. One way of doing this is to include items assessing participants' emotional responses to experiences of stigma. Six of the included measures (\#1, \#12-14, $\# 20$, \#21) contained such questions (e.g. 'After being teased, how upset were you?' (\#12), or 'I felt bad about my body' (\#20)). All of the adult measures (\#1,\#12-14) that included such items assessed emotional impact in connection with each question about stigma (for all four measures, participants rated 'how upset' each incident had made them on a 5-point Likert scale). In contrast, both of the child measures (\#20, \#21) assessed emotional impact separately, after assessing frequency of weight stigmatization. One measure asked children 'How did it (the experience with weight victimization) make you feel?' and had children rate feelings of anger and sadness as well as fear and effects on self-perception (e.g., 'I felt worse about myself') (\#20). The other measure assessed how the experience 'changed the way you feel about yourself?' (\#21) for general weight-related victimization as well as victimization from boys and girls separately.

\section{Modified Measures}

A number of the existing measures that assess experiences of weight stigma have been modified from previous scales for this purpose. Eleven of the 22 scales included in this review were adaptations or modifications of other scales (\#1-5, \#8,\#9, \#12-14, \#16). Four measures (\#3-5, \#8) were modified from scales measuring other types of stigma (e.g., gender, race, HIV). The other 7 measures were modifications of original scales assessing experiences with weight stigma, which were also included in this review. Perhaps not surprisingly, the most commonly cited scales were also the most modified. Three measures were adaptations of the Perceptions of Teasing Scale (POTS) (\#13, \#14, \#16) (itself a modification of the Perceptions of Appearance Related Teasing Scale (\#11)) and two were modifications of the Stigmatizing Situations Inventory (SSI) (\#1, \#9). Finally, one was a modification of the Family Teasing and Criticism (FAMTEASE) (\#2). 
Most commonly, measures were modified in order to specify or change the sources of stigma assessed. The weight teasing subscale of the POTS, which surveys teasing from general sources, was modified to reflect teasing from family members and peers (\#13, \#14, respectively), and the FAMTEASE scale was changed from assessing teasing from family members to teasing and criticism from a romantic partner (\#2). In addition, one scale modified the general teasing items on the POTS to examine teasing in physical activity settings (\#16). Other modifications involved changing the time period during which stigma was experienced, or the rating scale format. For example, one modified scale changed the time frame of stigma assessed by the SSI (of which the original scale assesses lifetime experiences of stigma), including three subscales measuring stigmatization in childhood, adolescence and adulthood separately (\#1), and added questions assessing the emotional impact of the perceived stigma, similar to those included on the POTS. Another measure changed the scale of the SSI from a 10-point to a 4-point frequency scale and divided the items into 11 subscales (\#9). Finally, the POTS (\#12) was created as a revision and update to the Physical Appearance Related Teasing Scale (PARTS) (\#11), changing the wording of the weight subscale to better assess stigma related to overweight.

\section{Strengths of Existing Measures}

Despite the general lack of research examining weight-related stigma from the perspective of the target, 22 measures met the relatively stringent inclusion criteria for this review, showing positive advancement in this area of study. While subject to limitations, there are clear advantages of using self-report survey methodology to measure a construct such as weight-related teasing or discrimination. Most obvious is the feasibility to construct, distribute, collect, and analyze responses from a survey compared to observational or qualitative data. All of the measures included in this review had acceptable internal consistencies, with Cronbach's $\alpha$ over 0.7 , and those that had been used in other samples exhibited similar consistencies, indicating that they each reliably assessed one construct, and almost all measures showed face validity for the examined construct (weight stigma). Thus, these scales are likely to be more useful as tools to measure self-reported weight stigmatization than single-item measurement.

Another strength of using survey methodology is that these existing measures allow data to be collected anonymously. This may be important in assessment of weight stigmatization, since many individuals who have experienced stigmatization may feel shame and embarrassment [46] and might not be as forthcoming in other modes of data collection that require face-to-face contact or do not protect anonymity.

Although a few measures specifically assess experiences with victimization related to excess weight, a number of them use 'weight' as the descriptor, which allows them to be used with samples of different weight categories. This is important in that it helps identify the extent to which weight stigmatization is experienced at different body weights, and how the nature and impact of this differs across weight categories.

Furthermore, six of the measures assess both the frequency of experienced weight victimization and its impact. This is important, as it allows us to begin to identify how different types of stigmatizing experiences affect individuals' emotions and behaviors. With increasing research showing links between experiences of stigma and negative psychological and physical health outcomes [47], it is pertinent to increase our understanding in this area and identify how different types of stigmatizing experiences impact health.

Given that weight stigmatization occurs in multiple settings, it is also notable that four of the measures examined experiences with weight bias across a variety of settings and 
sources (\#1, \#7, \#9, \#10). These may be particularly useful for researchers interested in a comprehensive assessment of experiences of weight stigmatization, and allow for examination of how experiences differ across multiple settings and perpetrators.

\section{Limitations of Existing Measures}

\section{Sampling}

There are a number of limitations regarding the samples used to validate existing measures. First, there is a significant lack of racial and ethnic diversity (which is rarely noted), and a number of these measures, even those intended or later appropriated for general use (\#12), have been assessed only in women (none were examined in all-male samples). This may represent a problem for generalizability given recent research that demonstrates gender differences in experiences of weight stigmatization [48-50] and potential differences for non-white individuals [51-53]. More work is needed to determine if there are qualitative differences in the experiences and attitudes of these groups, which requires existing measures to be tested in more diverse samples.

Additionally, although most existing measures were developed to assess experiences of stigmatization associated with excess weight, a number of these measures were instead assessed in participant samples where normal-weight individuals were overrepresented, or body weight was not reported, which makes results difficult to interpret. This limitation indicates the need for existing measures to be thoroughly assessed in samples comprised of overweight and obese individuals as well as of other body weight categories.

\section{Types of Stigma}

Given that the current review found only four measures that assessed experiences of weight stigma in a range of settings, there is a need for more comprehensive measures that assess multiple forms and sources of weight stigmatization. Most of the existing measures examine weight bias in interpersonal interactions, often in the context of verbal commentary related to the appearance of being overweight. However, there are many stereotypes (e.g., related to personality, character, and competence) specific to overweight and obese persons that lead to weight discrimination. Thus, it is important to separate physical appearance from more specific weight-related stigmatization, as the latter may lead to a different type of experience, particularly in employment and health care settings [54]. Measures that assess general weight stigmatization (rather than weight-based stigmatization in specific settings such as health care or during physical activity) miss important and informative details that can only be obtained with assessment that incorporates a variety of forms and sources of weight stigmatization.

In this context, it is important for measures to clarify definitions and descriptions of stigmatization, which can encompass a variety of experiences. For example, measures that aim to assess 'weight-related teasing,' may include a range of experiences ranging from fat jokes and name calling to serious verbal threats. Similarly, it may be important to distinguish experiences of teasing from bullying, which usually includes a power differential, refers to repeated behaviors [55], and may encompass a range of nonverbal behaviors and physical aggression not necessarily reflected in teasing. Furthermore, with increasing recognition of multiple forms of cyber bullying among youth, there is a need to ensure that this form of bullying is assessed in the context of body weight.

In addition, discrimination may also take different forms and include unfair treatment such as being fired from a job because of weight, being assigned lower wages compared to a thinner employee, or being denied appropriate medical treatment. Thus, it is important to 
broaden the focus of existing assessment from interpersonal interactions to include discriminatory policies such as unfair weight restrictions for certain jobs and BMI penalties on insurance premiums, as well as structural discrimination in our societal environment, including the lack of appropriately sized medical equipment for obese patients or inadequate accommodation in public modes of transportation, which can also be considered forms of stigma.

\section{Sources of Stigma}

Given the tendency of existing measures to focus on discrimination from general sources, it is important to increase the specificity regarding sources of weight stigma, as victimization from a stranger compared to a friend or loved one may have a different impact. Furthermore, assessing stigma from numerous sources will provide a more comprehensive understanding of a person's experience, as research has shown that stigma from multiple (compared to a single) sources may lead to worse outcomes [5].

In addition, it is important to further differentiate within the source categories that are currently used. For example, victimization from 'family members' may have a different impact if it consists of stigmatizing comments from a parent compared to teasing from a sibling, and peer victimization may be quite different if the perpetrator is a friend rather than a classmate.

It may also be helpful to place experiences of weight stigmatization in a broader context of other forms of stigma. For example, if a study aims to assess experiences of weight stigmatization among ethnic minorities, it may be useful to assess whether these experiences are more or less common (or impactful) than experiences of racial stigmatization, and how these experiences compare to (and interact with) stigma that may be experienced in other domains such as gender, sexual orientation, and disability.

\section{Weight Language}

There was considerable variability in the terminology used to refer to weight in the sample of measures, with some even switching between terms and using several descriptors for body weight within the same survey. Using subjective terms like 'heavy' and 'overweight' may be problematic, as these may perceived differently by different people and may not be very informative if they are administered in the absence of obtaining weight and height of respondents (which was not uncommon with measures using this terminology). Additionally, research suggests that words such as 'obese' or 'fat' may be perceived as stigmatizing [56-59], which could lead to individuals being less willing to claim this identity or may affect their responses on measures using this terminology.

Instead, it seems beneficial for measures to simply refer to 'weight' in survey items, which is a neutral descriptor, and to additionally collect self-reported height and weight of participants to calculate their BMI or weight category. Using 'weight' as the primary anchor in survey measures additionally allows experiences of weight-related stigma to be compared across participant weight categories, which may be particularly useful in light of research demonstrating that weight-related victimization may have harmful effects in normal-weight individuals as well [5].

\section{Time Frame}

Finally, the time frames of these measures should be given careful consideration. If participants are asked about lifetime experiences of stigmatization, recall bias may lead to an undue focus on recent instances of victimization. Measures that focus on experiences in childhood may overlook more relevant recent experiences, and may have different significance for individuals (e.g., for those who were overweight as children but have since reduced 
Table 3. Areas in need of empirical research to improve self-reported assessment of weight stigmatization

- Validation of self-report measures in wider range of demographic groups

- Development of measurement items that are relevant and sensitive to potential cultural differences in experiences of weight stigmatization

- Validation of self-report measures across a range of body weight categories

- Improved clarification of definitions and descriptors used to assess weight stigmatization (e.g., 'teasing' versus 'bullying' versus 'stigma' versus 'discrimination', etc.)

- Increased specification of terminology used to refer to body weight in existing measures (e.g., 'weight' versus 'overweight' versus 'obese' versus 'fat' versus 'heavy', etc.)

- Adaptation and validation of self-report measures assessing weight stigmatization from a range of sources (e.g. teachers, doctors, employers, family members, etc.)

- Increased specification of items assessing stigmatization from different source categories (e.g., 'family members' versus 'mother', 'father', 'siblings', 'grandparents', etc.)

- Development of measures that assess a wider variety of weight-related stigmatizing experiences and settings where these occur (e.g. indirect, environmental, institutional)

- Improved clarification of measurement items to distinguish between assessment of physical appearance stigmatization versus more specific weight-based stigmatization

- Adaptation and validation of measures to assess stigmatization across different time periods (e.g., during childhood, during the past year, lifetime discrimination, etc.)

- Development of self-report measures that assess both the nature and impact (e.g., emotional and behavioral responses) of experiences of weight stigmatization.

their BMI to the normal range compared to adults who have maintained their overweight status). Conversely, measures that focus only on stigmatizing experiences during the last year may overlook both recent and remote experiences that did not occur during this time frame. While it may be challenging to include assessment of different time periods in a single survey, consideration should be given to these limitations when selecting which time period of stigmatization to assess.

\section{Limitations, Conclusion and Directions for Future Research}

Several limitations of this review should be noted. While a thorough review of the relevant literature was conducted over the course of several months, it is possible that we missed articles containing measures that would otherwise have met the criteria for the review. Also, although the Cronbach's $\alpha$ for the measures included in the present study appear to meet general conventions of what constitutes 'adequate' (e.g., 0.70) or 'good' (e.g., 0.80 ) internal consistency, the use of cut-off values may be short-sighted if a measure having obtained such values is used without further consideration of its dimensionality or construct validity. [60] Therefore, we must interpret these numbers with caution. In addition, an analysis of multiple psychometric parameters and forms of measurement validity was not feasible to conduct in this review. This was primarily due to the absence of multiple studies assessing existing measures, a lack of theory-driven measurement in many studies, and the fact that most measures were either tested without comparison to other measures or were tested in conjunction with non-overlapping measures across studies. This presented considerable obstacles in the ability to make conclusions about the predictive and construct validity of existing measures. It will be important for future research to design adequate testing of existing measures so that their psychometric properties can be more clearly established. Finally, this review examined only measures of stigma due to excess weight, 
and the majority of adult assessments included in this study are appropriate only for use in overweight samples. However, it is important to acknowledge that individuals who are underweight may face stigma as well. Nonetheless, this study provides a comprehensive summary of existing measures of experiences with weight stigma.

Self-perceived weight discrimination is relatively a recent avenue of research, although the effects of this are now being studied in numerous domains. However, our knowledge in this area is only as good as the measures we have to study this phenomenon. The existing measures available to assess this construct contain a number of limitations and have been assessed in samples lacking diversity. Improvements in measurement are needed to achieve a clearer understanding of the nature and extent of self-perceived weight stigmatization and to develop measures that accurately reflect this type of victimization. Given these limitations, future researchers should draw upon existing research to extend and refine current scales to include more specific and varied experiences and sources of stigma, to (where possible) assess the emotional responses elicited by these experiences, to differentiate between appearance and weight, and to validate measures in variable and representative populations. Table 3 outlines specific directions for future research that will help improve measurement of self-perceived weight stigmatization and advance this area of study.

With high prevalence rates of obesity in multiple countries (including two thirds of Americans now being overweight or obese [61]), vast numbers of people stand to be affected by stigmatization and discrimination because of their weight. Despite widespread prejudice towards this population [48], many questions remain regarding the nature, extent, and impact of weight-based stigmatization experienced by so many. These important questions can only be adequately addressed with improved assessment in this area.

\section{Acknowledgements}

This research was supported by a grant from the Rudd Foundation.

\section{Disclosure Statement}

The authors declared no conflicts of interest.

\section{References}

1 Puhl RM, Heuer CA: The stigma of obesity: a review and update. Obesity 2009;17:941-964.

- 2 Puhl RM, Brownell KD: Bias, discrimination, and obesity. Obes Res 2001;9:788-805.

- 3 Crandall CS, D’Anello S, Sakalli N, Lazarus E, Nejtardt GW, Feather NT: An attribution-value model of prejudice: anti-fat attitudes in six nations. Pers Soc Psychol Bull 2001;27:30-37.

- 4 Andreyeva T, Puhl RM, Brownell KD: Changes in perceived weight discrimination among Americans: 19951996 through 2004-2006. Obesity 2008;16:1129-1134.

- 5 Eisenberg ME, Neumark-Sztainer D, Story M: Associations of weight-based teasing and emotional wellbeing among adolescents. Arch Pediatr Adolesc Med 2003;157:733-738.

- 6 Puhl RM, Moss-Racusin CA, Schwartz MB: Internalization of weight bias: implications for binge eating and emotional well-being. Obesity 2007;15:19-23.

7 Hayden-Wade HA, Stein RI, Ghaderi A, Saelens BE, Zabinski MF, Wilfey DE: Prevalence, characteristics, and correlates of teasing experiences among overweight children vs. non-overweight peers. Obes Res 2005;13: 1381-1392.

8 Carels RA, Wott CB, Young KM, Gumble A, Koball A, Oehlhof MW: Implicit, explicit, and internalized weight bias and psychosocial maladjustment among treatment-seeking adults. Eat Behav 2010;11:180-185.

9 Rudolph CW, Wells CL, Weller MD, Baltes BB: A meta-analysis of empirical studies of weight-based bias in the workplace. J Vocat Behav 2009;74:1-10. 
DePierre et al.: Experiences of Weight Stigmatization: A Review of Self-Report

10 Teachman BA, Brownell KD: Implicit anti-fat bias among health professionals: is anyone immune? Int J Obes Relat Metab Disord 2001;25:1525-1531.

11 Wardle J, Volz C, Golding C: Social variation in attitudes to obesity in children. Int J Obes Relat Metab Disord 1995;19:562-569.

12 Holub SC, Tan CC, Patel SL: Factors associated with mothers' obesity stigma and young children's weight stereotypes. J Appl Dev Psychol 2011;32:118-126.

13 Ruggs EN, King EB, Hebl M, Fitzsimmons M: Assessment of weight stigma. Obes Facts 2010;3:60-69.

14 Krukowski RA, Smith West D, Siddiqui NJ, Bursac Z, Phillips MM, Raczynski JM: No change in weight-based teasing when school-based obesity policies are implemented. Arch Pediatr Adolesc Med 2008;162:936942.

15 Sharma S, Wharton S, Forhan M, Kuk JL: Influence of weight discrimination on weight loss goals and selfselected weight loss interventions. Clin Obes 2011;1:153-160.

16 Carr D, Friedman MA: Is obesity stigmatizing? Body weight, perceived discrimination, and psychological well-being in the united states. J Health Soc Behav 2005;46:244-259.

17 Menzel JE, Schaefer LM, Burke NL, Mayhew LL, Brannick MT, Thompson JK: Apperance-related teasing, body dissatisfaction, and disordered eating: a meta-analysis. Body Image 2010;7:261-270.

18 Cash TF: Developmental teasing about physical appearance: Retrospective descriptions and relationships with body image. Soc Behav Pers 1995;23:123-130.

-19 Friedman KE, Ashmore JA, Applegate KL: Recent experiences of weight-based stigmatization in a weight loss surgery population: psychological and behavioral correlates. Obesity 2008;16(suppl 2):S69-S74.

20 Jones DC: Body image among adolescent girls and boys: a longitudinal study. Dev Psychol 2004;40:823835.

-21 Wonderlich SA, Crosby RD, Mitchell JE, Roberts JA, Haseltine B, DeMuth G, Thompson KM: Relationship of childhood sexual abuse and eating disturbance in children. J Am Acad Child Adolesc Psychiatry 2000;39: 1277-1283.

-22 Abraczinskas M, Fisak B, Barnes RD: The relation between parental influence, body image, and eating behaviors in a nonclinical female sample. Body Image 2012;9:93-100.

-23 Annis NM, Cash TF, Hrabosky JI: Body image and psychosocial differences among stable average weight, currently overweight, and formerly overweight women: the role of stigmatizing experiences. Body Image 2004;1:155-167.

24 Befort C, Robinson Kurpius SE, Hull-Blanks E, Nicpon MF, Huser L, Sollenberger S: Body image, self-esteem, and weight-related criticism form romantic partners. J Coll Stud Dev 2001;42:407-419.

-25 Carr D, Friedman MA, Jaffe K: Understanding the relationship between obesity and positive and negative affect: the role of psychosocial mechanisms. Body Image 2007;4:165-177.

-26 Farrow CV, Tarrant M: Weight-based discrimination, body dissatisfaction and emotional eating: the role of perceived social consensus. Psychol Health 2009;24:1021-1034.

$\checkmark 27$ Hatzenbuehler ML, Keyes KM, Hasin DS: Associations between perceived weight discrimination and the prevalence of psychiatric disorders in the general population. Obesity 2009;17:2033-2039.

-28 Kolotkin RL, Crosby RD, Koloski KD, Williams GR: Development of a brief measure to assess quality of life in obesity. Obes Res 2001;9:102-111.

-29 Myers A, Rosen JC: Obesity stigmatization and coping: Relation to mental health symptoms, body image, and self-esteem. Int J Obes Relat Metab Disord 1999;23:221-230.

-30 Polk DM, Hullman GA: Weight-related stigma as a predictor of self-disclosure patterns in women. Open Communication Journal 2011;5:1-10.

-31 Puhl RM, Brownell KD: Confronting and coping with weight stigma: an investigation of overweight and obese adults. Obesity 2006;14:1802-1815.

-32 Rand CW, MacGregor AM: Morbidly obese patients' perceptions of social discrimination before and after surgery for obesity. South Med J 1990;83:1390-1395.

-33 Thompson JK, Fabian LJ, Moulton DO, Dunn ME, Altabe MN: Development and validation of the physical appearance related teasing scale. J Pers Assess 1991;56:513-521.

-34 Thompson JK, Cattarin J, Fowler B, Fisher E: The perception of teasing scale (POTS): a revision and extension of the physical appearance related teasing scale (parts). J Pers Assess 1995;65:146-157.

-35 van den Berg P, Thompson JK, Obremski-Brandon K, Coovert M: The tripartite influence model of body image and eating disturbance. A covariance structure modeling investigation testing the mediational role of appearance comparison. J Psychosom Res 2002;53:1007-1020.

-36 Wadden TA, Anderson DA, Foster GD, Bennet A, Steinberg C, Sarwer DB: Obese women's perceptions of their physician's weight management attitudes and practices. Arch Fam Med 2000;9:854-860.

-37 Faith MS, Leone MA, Ayers TS, Moonseong H, Pietrobelli A: Weight criticism during physical activity, coping skills, and reported physical activity in children. Pediatrics 2002;110:e23-e31.

-38 Kolotkin RL, Zeller MH, Modi AC, Samsa GP, Polanichka Quinlan N, Yanovski JA, Bell SK, Maahs DM, Gonzales de Serna D, Roehrig HR: Assessing weight-related quality of life in adolescents. Obesity 2006;14:448-457.

-39 Levine MP, Smolak L, Hayden H: The relation of sociocultural factors to eating attitudes and behaviors among middle-school girls. J Early Adolesc 1994;14:471-490.

40 Oliver KK, Thelen MH: Children's perceptions of peer influence on eating concerns. Behav Ther 1996;27: 25-39. 
41 Puhl RM, Luedicke J, Heuer C: Weight-based victimization toward overweight adolescents: observations and reactions of peers. J Sch Health 2011;81:696-703.

42 Shisslak CM, Renger R, Sharpe T, Crago M, McKnight KM, Gray N, Bryson S, Estes LS, Parnaby OG, Killen J, Taylor CB: Development and evaluation of the mcknight risk factor survey for assessing potential risk and protective factors for disordered eating in preadolescent and adolescent girls. Int J Eat Disord 1999;25: 195-214.

-43 Young-Hyman D, Schlundt DG, Herman-Wenderoth L, Bozylinski K: Obesity, appearance, and psychosocial adaptation in young African American children. J Pediatr Psychol 2003;28:463-472.

44 National Heart, Lung, and Blood Institute. How are overweight and obesity diagnosed? 2010. Available at http://www.nhlbi.nih.gov/health/health-topics/topics/obe/diagnosis.html (last accessed December 6, 2012).

-45 Williams DR, Neighbors HW, Jackson JS: Racial/ethnic discrimination and health: findings from community studies. Am J Public Health 2003;93:200-208.

$\checkmark 46$ Lewis S, Thomas SL, Blood RW, Castle DJ, Hyde J, Komesaroff PA: How do obese individuals perceive and respond to the different types of obesity stigma that they encounter in their daily lives? A qualitative study. Soc Sci Med 2011;73:1349-1356.

47 Puhl RM, Heuer CA: Obesity stigma: important considerations for public health. Am J Public Health 2010; 100 1019-1028.

48 Roehling MV, Roehling PV, Pichler S: The relationship between body weight and perceived weight-related employment discrimination: the role of sex and race. J Vocat Behav 2007;71:300-318.

49 Puhl RM, Andreyeva T, Brownell KD: Perceptions of weight discrimination: Prevalence and comparison to race and gender discrimination in America. Int J Obes (Lond) 2008;32:992-1000.

50 Puhl RM, Heuer C, Sarda V: Framing messages about weight discrimination: impact on public support for legislation. Int J Obes (Lond) 2010:1-10.

-51 Carr D, Jaffe KJ, Friedman MA: Perceived interpersonal mistreatment among obese Americans: do race, class and gender matter? Obesity 2008;16(suppl 2):S60-S68.

52 Hebl MR, Turchin JM: The stigma of obesity: what about men? Basic Appl Soc Psych 2005;27:267-275.

$\$ 53$ Perez-Lopez MS, Lewis RJ, Cash TF: The relationship of antifat attitudes to other prejudicial and genderrelated attitudes. J Appl Soc Psychol 2001;31:683-669.

54 Puhl RM, Peterson JL: Physical appearance and stigma; in Cash T (ed): Encyclopedia of Body Image and Human Appearance, Vol 2. San Diego, Academic Press; 2012, pp 588-594.

55 Olweus D: Knowlege base and an effective intervention program. Ann N Y Acad Sci 2006;794:265-276.

56 Vartanian LR: 'Obese people' vs 'fat people': impact of group label on weight bias. Eat Weight Disord 2010; 15:e195-e198.

57 Puhl RM, Peterson JL, Luedicke J: Motivating or stigmatizing? Public perceptions of language about weight used by health providers. Int J Obes (Lond) 2012; doi: 10.1038/ijo.2012.110.

58 Puhl RM, Peterson JL, Luedicke J: Parental perceptions of weight terminology that providers use with youth. Pediatrics 2011;128:e786-e793.

59 Brochu PM, Esses VM: What's in a name? The effects of the labels 'fat' versus 'overweight' on weight bias. J Appl Soc Psychol 2011;41:1981-2008.

60 Schmitt N: Uses and abuses of coefficient alpha. Psychol Assess 1996;84:350-353.

61 Flegal KM, Carroll MD, Kit BK, Ogden CL. Prevalence of obesity and trends in the distribution of body mass index among US adults, 1999-2010. JAMA 2012;307:E1-E7.

62 Puhl RM, Luedicke J: Weight-based victimization among adolescents in the school setting: emotional reactions and coping behaviors. J Youth Adolesc 2012;41:27-40. 